\title{
The Long-Term Outcome of CyberKnife-Based Stereotactic Radiotherapy for Intra/ Extracranial Non-Vestibular Schwannomas: A Single-Center Experience
}

Sukwoo Hong ( $\square$ honsohkaisei6031@gmail.com )

University of Tokyo https://orcid.org/0000-0002-3664-9245

Kenji Kagawa

University of Tokyo

Kengo Sato

University of Tokyo

Ryutaro Nomura

Chigasaki Chuo Hospital

Shunsuke Ichi

University of Tokyo

\section{Research Article}

Keywords: CyberKnife, non-vestibular, schwannoma, hypofractionated, fractionation

Posted Date: January 3rd, 2022

DOI: https://doi.org/10.21203/rs.3.rs-1078736/v2

License: (c) (i) This work is licensed under a Creative Commons Attribution 4.0 International License.

Read Full License 


\section{Abstract}

The long-term outcomes of CyberKnife-based hypofractionated stereotactic radiotherapy (SRT) for intra/ extracranial non-vestibular schwannomas (nVS) need to be accumulated. Patients who received SRT by CyberKnife for nVS from 2010 to 2019 were retrospectively reviewed. A total of 45 patients with nVS were identified. The mean age was $53( \pm 18)$ years old, and 23 patients $(51 \%)$ were female. Twenty-nine patients $(64 \%)$ had previous procedures. As for the tumor extension, $22(49 \%) n V S$ were classified as primary intracranial, five (11\%) were classified as intra/ extracranial (dumbbell shape), and $18(40 \%)$ were classified as primary extracranial. The median prescribed dose, covering $95 \%$ of the planning target volume, was 21 (IQR $21-25) \mathrm{Gy}$, and the median target volume was 7 (IQR 3.6-13.1) $\mathrm{cm}^{3}$. The local control rate of $\mathrm{nVS}$ for patients without neurofibromatosis type 2 (NF2) was $100 \%$. Old age (OR 0.92 , pvalue 0.03 ) and previous surgery (OR 0.02 , p-value 0.02 ) were significant risk factors for no symptomatic improvement. The progression-free survival was $74( \pm 33)$ months clinically and 69 (IQR $36-94)$ months radiologically. During follow-up, two cases (4\%) with NF2 resulted in treatment failure, 13 cases $(41 \%)$ resulted in transient tumor expansion (TTE), 10 (22\%) suffered from transient adverse radiation effect (ARE), and two (4\%) resulted in permanent ARE. Hypofractionated SRT by CyberKnife for head, neck, and spine nVS was an effective treatment regardless of tumor extension relative to the cranium. Although the risk of permanent ARE was low, some patients experienced transient clinical worsening due to TTE.

\section{Introduction}

Despite the progressive advancement of surgical armamentarium, schwannomas in the head, neck, and spine may be difficult to treat because of proximity to nerves and arteries.[16, 12] They are World Health Organization grade I benign tumors and commonly arise outside the central nervous system. Most intracranial schwannomas arise from the vestibular nerve, and schwannomas from other cranial nerves are not frequently encountered. Intracranial and spinal schwannomas are treated by either or combinations of microsurgical resection, stereotactic radiosurgery (SRS), and stereotactic radiotherapy (SRT).[2] To date, compared to the data on SRS outcome on vestibular schwannomas, we have less data on non-vestibular schwannomas (nVS). And since most of the radiation therapy is performed by gamma knife[6, 5], we have even fewer data of SRT performed by CyberKnife, especially on nVS.

CyberKnife can treat lesions outside as well as inside the cranium, which is a key difference from gamma knife. Some schwannomas around the skull base foramina or canals, such as jugular foramen and hypoglossal schwannomas, present with lesions occupying both inside and outside the cranium (dumbbell shape) or primarily extracranial regions. These tumors are theoretically more suitable for CyberKnife than gamma knife.

Our facility is a referral center for CyberKnife, and we have treated quite a few nVS occupying extracranial spaces with or without intracranial space. Considering the rarity of SRT outcome on nVS, we thought our data might add further evidence to the current literature. 


\section{Methods}

\section{Patient selection}

Consecutive patients who received SRT for nVS in the head, neck, and spine between 2010 and 2019 were included. Of note, since the authors' hospital is a referral center for CyberKnife therapy, most of the included patients were referred from different hospitals and heterogeneous in baseline characteristics. Various data were recorded, including age, sex, symptoms at the time of SRT, history of previous operation or radiation, the status of neurofibromatosis type 2 (NF2), the form of treatment (upfront, adjuvant, upfront for relapsed cases, and adjuvant for relapsed cases), parameters for SRT, radiological response, changes in symptoms, adverse radiation effect (ARE), and length of follow-up, among others. The diagnosis of schwannoma was made radiologically by MRI for non-operated patients and further confirmed pathologically for operated patients. There were no strict criteria for surgical resection, as the patients were referred from various hospitals. However, surgery tends to have been performed for younger patients. Postoperative SRT was recommended for those with residual tumors. The tumor locations were classified into the following three types relative to the cranium: primary intracranial, intra/ extracranial (dumbbell shape), and primary extracranial. This is the result of modifying the classification described by Martin et al[8]

\section{SRT at our facility}

Target volumes were delineated on thin-slice CT with or without gadolinium-enhanced MRI. A 6D-skull tracking algorithm was used. In planning treatment, we selected the prescription dose and fractionation according to the size and site of the lesions based on the senior authors' (K.S. and R.N.) experience. Most of the tumors were treated $21 \mathrm{~Gy}$ over 3 fractions or $25 \mathrm{~Gy}$ over 5 fractions.

\section{Patient evaluation and tumor response}

Based on the MRI, we classified the response to SRT into two categories of under control and treatment failure, like a previous study[16] using perpendicular diameters as reference. The senior authors (K.S. and R.N.) classified tumor response into the two categories, with confirmation by the primary author (S.H.). Adverse radiation effect (ARE) was evaluated by Common Terminology Criteria for Adverse Events (CTCAE), if listed.[10] Transient tumor expansion (TTE), which is known to be a common phenomenon after SRS to vestibular schwannoma[9], and concurrent clinical changes were recorded and counted as ARE if clinically symptomatic.

\section{Statistical analysis}

From the acquired data, univariate and multivariate analyses were performed to identify risk factors for symptom improvement. A multicollinearity test was done to identify factors to exclude in multivariate analysis. Statistical analysis was done using SPSS version 25.0 (IBM Inc., Armonk, NY, USA). Values were listed as means for parametric data, and median for nonparametric data. The Shapiro-Wilk test was used 
to differentiate parametric from nonparametric data. Binary logistic regression was used to evaluate risk factors for symptom improvement. A p-value of 0.05 or less was considered statistically significant.

Our institutional review board did not require informed consent for study participation because this study relied on information obtained as part of routine clinical practice.

\section{Results}

\section{Baseline characteristics}

A total of 45 schwannomas in 45 patients were identified (Table 1). Females constituted $51 \%$ of the patients. Common presenting signs and symptoms at the time of SRT were diplopia, facial dysesthesia, trigeminal neuralgia, dysphagia, dysarthria, hoarseness, and radiculopathy depending on the tumor location and previous surgical complications. Of the five (11\%) patients who had no symptoms, three patients received SRT for enlarging tumor size after wait and scan, one patient received the treatment for relapse, and the other one received for residual tumor after surgery. Most common tumor were trigeminal schwannomas (27\%). A previous operation had been performed in 29 cases $(64 \%)$. No cases had a previous history of radiation treatment. Two cases (4\%) were neurofibromatosis type 2 . The most common form of SRT was upfront treatment for newly-diagnosed tumors (18 cases, $40 \%$ ), followed by upfront treatment for relapsed tumors ( 16 cases, 36\%) and adjuvant treatment ( 9 cases, $20 \%$ ). The median time between the previous surgery and SRT was 2 months and that for the relapsed tumor was about 35 months (Table 1). 
Table 1

Summary of the baseline patient characteristics.

\begin{tabular}{|ll|}
\hline Total & 45 \\
\hline Age (mean) (yr) & $53 \pm 18$ \\
\hline Female & $23(51 \%)$ \\
\hline Previous operation & $29(64 \%)^{\star}$ \\
Previous radiation & $0(0 \%)$ \\
\hline NF2 & $2(4 \%)$ \\
\hline Tumor base & \\
Trigeminal nerve (V) & $12(26.7 \%)$ \\
Abducens nerve (VI) & $1(2.2 \%)$ \\
Facial nerve (VII) & $3(6.7 \%)$ \\
Lower cranial nerves (IX - XI) & $13(28.9 \%)$ \\
Hypoglossal nerve (XII) & $8(17.8 \%)$ \\
Spine & $6(13.3 \%)$ \\
Parapharyngeal space & $2(4.4 \%)$ \\
\hline Form of hSRT & \\
Upfront & $18(40.0 \%)^{\star}$ \\
Adjuvant & $9(20.0 \%)$ \\
Upfront for relapsed & $16(35.6 \%)$ \\
Adjuvant for relapsed & $2(4.4 \%)$ \\
\hline Time from the last surgery to hSRT (median) (IQR) & $2(1-5)$ mos \\
Time from the last surgery to relapse (median) (IQR) & $35(13-83)$ mos \\
\hline tTwo patients who underwent biopsy were counted as operated and classified into the upfront \\
\hline treatment group. \\
\hline The
\end{tabular}

The parameters of SRT are summarized in Table 2. Most therapies were done in multiple sessions, and the median target tumor volume was $7(3.6-13.1) \mathrm{cm}^{3}$. Relative to the cranium, $51 \%$ of tumors had extracranial tumor portions (Table 3). We illustrate two cases as examples (Fig.). 
Table 2

The parameters of hypofractionated stereotactic radiotherapy. The parametric data are represented in the mean value ( \pm standard deviation), whereas nonparametric data are represented in the median value (interquartile range). Roman numerals represent respective numbered cranial nerves. Since we had only two parapharyngeal space schwannomas, we showed the exact values in the column.

\begin{tabular}{|c|c|c|c|c|c|c|c|c|}
\hline & Total & V & VI & VII & IX-XI & XII & Spine & $\begin{array}{l}\text { Parapharyngeal } \\
\text { space }\end{array}$ \\
\hline $\mathrm{D}_{95 \%}(\mathrm{~Gy})$ & $\begin{array}{l}21(21- \\
25)\end{array}$ & $\begin{array}{l}21 \\
(16.5- \\
24.5)\end{array}$ & 21 & $24( \pm 5)$ & $\begin{array}{l}21(21- \\
25)\end{array}$ & $\begin{array}{l}21 \\
( \pm 3)\end{array}$ & 21 & 25,28 \\
\hline Fraction & $3(3-5)$ & $3(2-5)$ & 3 & $5( \pm 2)$ & $5(3-5)$ & $3( \pm 2)$ & 3 & 5,7 \\
\hline $\begin{array}{l}\text { Target } \\
\text { diameter } \\
(\mathrm{mm})\end{array}$ & $\begin{array}{l}23(19- \\
29)\end{array}$ & $\begin{array}{l}21 \\
( \pm 10)\end{array}$ & 18 & $\begin{array}{l}30 \\
( \pm 18)\end{array}$ & $26( \pm 6)$ & $\begin{array}{l}24 \\
( \pm 6)\end{array}$ & $25( \pm 8)$ & 39,47 \\
\hline $\begin{array}{l}\text { Prescription } \\
\text { isodose (\%) }\end{array}$ & $79( \pm 4)$ & $80( \pm 4)$ & 85 & $\begin{array}{l}79(79- \\
80)\end{array}$ & $79( \pm 3)$ & $\begin{array}{l}81 \\
( \pm 4)\end{array}$ & $75( \pm 4)$ & 78,79 \\
\hline $\begin{array}{l}\text { Target } \\
\text { volume } \\
\left(\mathrm{cm}^{3}\right)\end{array}$ & $\begin{array}{l}7(3.6- \\
13.1)\end{array}$ & $\begin{array}{l}4.8 \\
(1.7- \\
10)\end{array}$ & 3.3 & $\begin{array}{l}27 \\
( \pm 40)\end{array}$ & $\begin{array}{l}7.7 \\
(6.7- \\
12.5)\end{array}$ & $\begin{array}{l}8.4 \\
( \pm 6.1)\end{array}$ & $\begin{array}{l}10 \\
( \pm 9.6)\end{array}$ & 33,55 \\
\hline $\begin{array}{l}\text { Target } \\
\text { covered } \\
\left(\mathrm{cm}^{3}\right)\end{array}$ & $\begin{array}{l}6.7 \\
(3.5- \\
12.5)\end{array}$ & $\begin{array}{l}4.6 \\
(1.7- \\
9.9)\end{array}$ & 3.1 & $\begin{array}{l}26 \\
( \pm 38)\end{array}$ & $\begin{array}{l}7.3 \\
(6.4- \\
12.1)\end{array}$ & $\begin{array}{l}8.1 \\
( \pm 5.9)\end{array}$ & $\begin{array}{l}10.1 \\
( \pm 9.1)\end{array}$ & 31,53 \\
\hline $\begin{array}{l}\text { Target } \\
\text { covered (\%) }\end{array}$ & $\begin{array}{l}95(95- \\
96)\end{array}$ & $\begin{array}{l}95(95- \\
96)\end{array}$ & 95 & $\begin{array}{l}95 \\
( \pm 0.5)\end{array}$ & $\begin{array}{l}95(95- \\
96)\end{array}$ & $\begin{array}{l}95 \\
(95- \\
96)\end{array}$ & $\begin{array}{l}95 \\
( \pm 0.5)\end{array}$ & 96,95 \\
\hline $\begin{array}{l}\text { Prescribed } \\
\text { isodose } \\
\text { volume/ } \\
\text { target } \\
\text { isodose } \\
\text { volume }\end{array}$ & $\begin{array}{l}1.31 \\
(1.21- \\
1.4)\end{array}$ & $\begin{array}{l}1.28 \\
(1.21- \\
1.36)\end{array}$ & 1.54 & $\begin{array}{l}1.32 \\
( \pm 0.09)\end{array}$ & $\begin{array}{l}1.29 \\
( \pm 0.11)\end{array}$ & $\begin{array}{l}1.27 \\
(1.2- \\
1.37)\end{array}$ & $\begin{array}{l}1.52 \\
( \pm 0.3)\end{array}$ & $1.17,1.31$ \\
\hline $\begin{array}{l}\text { New } \\
\text { conformity } \\
\text { index }\end{array}$ & $\begin{array}{l}1.37 \\
(1.27- \\
1.46)\end{array}$ & $\begin{array}{l}1.33 \\
(1.25- \\
1.43)\end{array}$ & 1.62 & $\begin{array}{l}1.38 \\
( \pm 0.09)\end{array}$ & $\begin{array}{l}1.35 \\
( \pm 0.12)\end{array}$ & $\begin{array}{l}1.32 \\
(1.25- \\
1.45)\end{array}$ & $\begin{array}{l}1.59 \\
( \pm 0.31)\end{array}$ & $1.22,1.36$ \\
\hline $\begin{array}{l}\text { Max dose/ } \\
\text { prescribed } \\
\text { dose }\end{array}$ & $\begin{array}{l}1.25 \\
( \pm 0.06)\end{array}$ & $\begin{array}{l}1.24 \\
( \pm 0.06)\end{array}$ & 1.18 & $\begin{array}{l}1.27 \\
(1.24- \\
1.27)\end{array}$ & $\begin{array}{l}1.26 \\
( \pm 0.06)\end{array}$ & $\begin{array}{l}1.22 \\
(0.06)\end{array}$ & $\begin{array}{l}1.32 \\
( \pm 0.07)\end{array}$ & $1.28,1.27$ \\
\hline
\end{tabular}


Table 3

Tumor classification based on its spatial relation to the cranium. Other than spinal schwannomas, $17(38 \%)$ schwannomas had extracranial tumor portion.

\begin{tabular}{|llllllll|}
\hline & Total & V, VI & VII & IX - XI & XII & Spine & Parapharyngeal space \\
\hline Primary intracranial & $22(49 \%)$ & 13 & 1 & 4 & 4 & 0 & 0 \\
\hline Intra/ extracranial & $5(11 \%)$ & 0 & 1 & 2 & 2 & 0 & 0 \\
\hline Primary extracranial & $18(40 \%)$ & 0 & 1 & 7 & 2 & 6 & 2 \\
\hline Total & 45 & 13 & 3 & 13 & 8 & 6 & 2 \\
\hline
\end{tabular}

\section{Outcome}

The treatment outcome is summarized in Table 4. Forty-three patients (96\%) were under control. Two NF2 patients $(4 \%)$ resulted in treatment failure. Symptomatic improvement was only apparent in eight patients $(20 \%)$ out of the 40 patients who were symptomatic at the time of SRT (Supplementary Table 1). Multivariate analysis revealed that older age (OR 0.92, p-value 0.03) and operated status (OR 0.01, pvalue 0.01 ) were significant risk factors for no symptomatic improvement (Supplementary Table 2). 
Table 4

Outcome after stereotactic radiotherapy. The parametric data are represented in the mean value ( \pm standard deviation), whereas nonparametric data are represented in the median value (interquartile range).

\begin{tabular}{|c|c|c|c|c|c|c|c|}
\hline & Total & $\mathrm{V}, \mathrm{VI}$ & VII & $\begin{array}{l}\text { IX- } \\
\text { XI }\end{array}$ & XII & Spine & $\begin{array}{l}\text { Parapharyngeal } \\
\text { space }\end{array}$ \\
\hline Under control & $\begin{array}{l}43 \\
(96 \%)\end{array}$ & 13 & 3 & 13 & 8 & 4 & 2 \\
\hline Treatment failure & $2(4 \%)$ & 0 & 0 & 0 & 0 & $2 *$ & 0 \\
\hline $\begin{array}{l}\text { Symptom } \\
\text { improvement** }\end{array}$ & $\begin{array}{l}8 / 40 \\
(20 \%)\end{array}$ & 2 & 0 & 2 & 2 & 1 & 1 \\
\hline $\begin{array}{l}\text { Transient tumor } \\
\text { expansion } \star \star \star\end{array}$ & $\begin{array}{l}13 / 32 \\
(41 \%)\end{array}$ & $3 / 10$ & $3 / 3$ & $2 / 5$ & $3 / 8$ & $1 / 4$ & $1 / 2$ \\
\hline ARE (transient) & $\begin{array}{l}10 \\
(22 \%)\end{array}$ & 2 & 2 & 3 & 2 & 1 & 0 \\
\hline ARE (permanent) & $2(4 \%)$ & 0 & 0 & 1 & 0 & 0 & 1 \\
\hline $\begin{array}{l}\text { PFS (mos) } \\
\text { (radiological) }\end{array}$ & $\begin{array}{l}69(36- \\
94)\end{array}$ & $\begin{array}{l}71 \\
( \pm 38)\end{array}$ & $\begin{array}{l}41(40.5 \\
-59)\end{array}$ & $\begin{array}{l}60 \\
( \pm 39)\end{array}$ & $\begin{array}{l}71 \\
( \pm 41)\end{array}$ & $\begin{array}{l}64 \\
( \pm 28)\end{array}$ & $93.5(92-95)$ \\
\hline PFS (mos) (clinical) & $74( \pm 33)$ & $\begin{array}{l}77 \\
( \pm 37)\end{array}$ & $71( \pm 29)$ & $\begin{array}{l}64 \\
( \pm 37)\end{array}$ & $\begin{array}{l}80 \\
( \pm 36)\end{array}$ & $\begin{array}{l}76 \\
( \pm 23)\end{array}$ & $93.5(92-95)$ \\
\hline
\end{tabular}

*NF2, upfront

**Excluded the five patients who were asymptomatic at the time of SRT

***Excluded 13 patients whose MRI data were not available

\section{Adverse effects}

Thirteen patients ( $41 \%$ ) out of 32 patients whose past MRI data were all available experienced TTE (Table 4). Among the 13 patients, eight patients $(25 \%, 8 / 32)$ were clinically symptomatic by headache or worsening of the original symptoms. These transient symptoms were all managed by supportive care with medicine or observation. Ten patients (22\%) experienced transient ARE. Eight patients were associated with TTE, one patient with IX-XI schwannoma had a transient hearing problem that persisted for about a year, and the other patient with IX-XI schwannoma experienced nausea, dizziness, and dysphagia. Two patients (4\%) resulted in permanent ARE. One patient with parapharyngeal space schwannoma resulted in dysgeusia (CTCAE grade 1), local muscle rigidity, and decreased salivation. The other patient with IX-XI schwannoma resulted in dysgeusia (CTCAE grade 1) and tinnitus (CTCAE grade 1).

\section{Discussion}


We showed that SRT effectively controlled nVS in the head, neck, and spine, regardless of the spatial relation to the cranium. As for non-NF2 patients, the local control rate was $100 \%$ in our cohort (Table 4). Although the severity and rate of permanent ARE were mild (CTCAE grade 1) and low (4\%), clinical worsening due to TTE should be kept in mind, as $25 \%$ resulted in transient symptomatic deterioration, which was controlled with or without medications. The comparison of the treatment result of our study population to the past studies is summarized in Table 5.[11, 14, 17, 3, 1, 13, 2, 15, 4, 12, 7, 5, 6, 16] Except for spinal schwannomas, we still have few long-term data on nVS treated by SRT. 
Table 5

Summary of past literature on stereotactic radiosurgery (SRS) or stereotactic radiotherapy (SRT) on nonvestibular schwannomas. Roman numerals represent respective numbered cranial nerves. Compared to SRS by gamma knife, fewer data exist regarding the outcome after SRT by Cyberknife (shown in italic font). Our study is a study with comparable cohort size with relatively longer follow-up among the few studies on CyberKnife.

\begin{tabular}{|c|c|c|c|c|c|c|}
\hline & $\begin{array}{l}\mathrm{N} \text { of } \\
\text { tumors }\end{array}$ & $\begin{array}{l}\text { Primary } \\
\text { treatment } \\
\text { modality }\end{array}$ & $\begin{array}{l}\text { Tumor location } \\
\text { based on the } \\
\text { nerves }\end{array}$ & $\begin{array}{l}f / u \\
\text { period }\end{array}$ & LCR & Clinical AE \\
\hline $\begin{array}{l}\text { Pan et al., } \\
2005 \text { [11] }\end{array}$ & 56 & $\begin{array}{l}\text { SRS by } \\
\text { Yknife }\end{array}$ & V & $\begin{array}{l}68 \text { mos } \\
\text { mean }\end{array}$ & $93 \%$ & Not described \\
\hline $\begin{array}{l}\text { Sheehan et al., } \\
2007 \text { [14] }\end{array}$ & 26 & $\begin{array}{l}\text { SRS by } \\
\text { Yknife }\end{array}$ & v & $\begin{array}{l}48.5 \\
\text { mos } \\
\text { mean }\end{array}$ & $88 \%$ & None \\
\hline $\begin{array}{l}\text { Showalter et } \\
\text { al., 2008 [17] }\end{array}$ & 39 & $\begin{array}{l}\text { SRS (15) } \\
\text { yknife/ } \\
\text { fSRT by } \\
\text { LINAC(24) }\end{array}$ & $\begin{array}{l}I I I(2), V(19), V I \\
\text { (3), VII (5), IX-XI } \\
\text { (7), XII (2), } \\
\text { cavernous sinus } \\
\text { (1) }\end{array}$ & $\begin{array}{l}24 \text { mos } \\
\text { median }\end{array}$ & $\begin{array}{l}95 \% \\
(2 y)\end{array}$ & $\begin{array}{l}\text { Cranial } \\
\text { neuropathy (4\%) }\end{array}$ \\
\hline $\begin{array}{l}\text { Hamm et al., } \\
2008 \text { [3] }\end{array}$ & 19 & $\begin{array}{l}\text { SRS/ } \\
\text { hSRT/ } \\
\text { cSRT by } \\
\text { LINAC }\end{array}$ & $\begin{array}{l}V(13), I X-X I(5), \\
\text { orbit (1) }\end{array}$ & $\begin{array}{l}35 \text { mos } \\
\text { median }\end{array}$ & $\begin{array}{l}88 \% \\
(5 y)\end{array}$ & None \\
\hline $\begin{array}{l}\text { Choi et al., } \\
2011 \text { [1] }\end{array}$ & 42 & $\begin{array}{l}\text { SRS, hSRT } \\
\text { by yknife }\end{array}$ & $\begin{array}{l}\text { IV }(1), \mathrm{V}(18), \text { VII } \\
(6), I X-X I(13), X I I \\
(2), \text { cavernous } \\
\text { sinus (2) }\end{array}$ & $\begin{array}{l}29 \text { mos } \\
\text { median }\end{array}$ & $98 \%$ & $\begin{array}{l}\text { Cranial } \\
\text { neuropathy (5\%) }\end{array}$ \\
\hline $\begin{array}{l}\text { Sachdev et al., } \\
2011[13]\end{array}$ & 47 & $\begin{array}{l}\text { SRS/ hSRT } \\
\text { by } \\
\text { CyberKnife }\end{array}$ & Spine & $\begin{array}{l}29 \text { mos } \\
\text { median }\end{array}$ & $98 \%$ & $\begin{array}{l}\text { Transient myelitis } \\
(2 \%)\end{array}$ \\
\hline $\begin{array}{l}\text { Elsharkawy et } \\
\text { al., 2012 [2] }\end{array}$ & 36 & $\begin{array}{l}\text { SRS by } \\
\text { Yknife }\end{array}$ & $\begin{array}{l}\text { III (1), IV (1), V } \\
(25), \mathrm{VI}(2), \mathrm{VII} \\
(1), \mathrm{IX}(1), \mathrm{XII}(3)\end{array}$ & $\begin{array}{l}54 \text { mos } \\
\text { mean, } \\
37 \text { mos } \\
\text { median }\end{array}$ & $\begin{array}{l}91 \% \\
(2 y)\end{array}$ & $\begin{array}{l}\text { Dysarthria/ } \\
\text { ambulation } \\
\text { worsening (3\%), } \\
\text { facial dysesthesia } \\
(6 \%)\end{array}$ \\
\hline $\begin{array}{l}\text { Shin et al., } \\
2015[15]\end{array}$ & 47 & $\begin{array}{l}\text { SRS by } \\
\text { LINAC }\end{array}$ & Spine & $\begin{array}{l}44 \text { mos } \\
\text { median }\end{array}$ & $95 \%$ & None \\
\hline $\begin{array}{l}\text { Puataweepong } \\
\text { et al., } 2016 \\
\text { [12] }\end{array}$ & 52 & $\begin{array}{l}\text { hSRT by X- } \\
\text { Knife (19) } \\
\text { and } \\
\text { CyberKnife } \\
\text { (33) }\end{array}$ & $\begin{array}{l}\text { II (3), III (2), V } \\
\text { (14), VI (1), VII } \\
\text { (9), IX-XI (20), XII } \\
\text { (2), cavernous } \\
\text { sinus (1) }\end{array}$ & $\begin{array}{l}36 \text { mos } \\
\text { median }\end{array}$ & $\begin{array}{l}94 \% \\
(3 y), \\
88 \% \\
(5 y)\end{array}$ & $\begin{array}{l}\text { Mass effect due } \\
\text { to tumor } \\
\text { expansion that } \\
\text { required surgical } \\
\text { intervention (6\%) }\end{array}$ \\
\hline
\end{tabular}

LCR: local control rate, y: year, mos: month, cSRT: conventional SRT, fSRT: fractionated SRT, hSRT: hypofractionated SRT, LINAC: linear accelerator 


\begin{tabular}{|c|c|c|c|c|c|c|}
\hline & $\begin{array}{l}\mathrm{N} \text { of } \\
\text { tumors }\end{array}$ & $\begin{array}{l}\text { Primary } \\
\text { treatment } \\
\text { modality }\end{array}$ & $\begin{array}{l}\text { Tumor location } \\
\text { based on the } \\
\text { nerves }\end{array}$ & $\begin{array}{l}f / u \\
\text { period }\end{array}$ & LCR & Clinical AE \\
\hline $\begin{array}{l}\text { Hasegawa et } \\
\text { al., } 2016 \text { [4] }\end{array}$ & 117 & $\begin{array}{l}\text { SRS by } \\
\text { yknife }\end{array}$ & IX-XI & $\begin{array}{l}52 \text { mos } \\
\text { median }\end{array}$ & $\begin{array}{l}91 \% \\
(3 y) \\
89 \% \\
(5 y)\end{array}$ & $\begin{array}{l}\text { Transient } \\
\text { symptomatic } \\
\text { deterioration } \\
\text { (10\%), persistent } \\
\text { deterioration (7\%) }\end{array}$ \\
\hline $\begin{array}{l}\text { Langlois et al., } \\
2018 \text { [7] }\end{array}$ & 35 & $\begin{array}{l}\text { SRS by } \\
\text { Yknife }\end{array}$ & $\begin{array}{l}\text { IV (1), V (20), VI } \\
\text { (2), VII (7), IX-XI } \\
\text { (5) }\end{array}$ & $\begin{array}{l}48 \text { mos } \\
\text { median }\end{array}$ & $\begin{array}{l}94 \% \\
(5 y) \\
88 \% \\
(10 y)\end{array}$ & $\begin{array}{l}21 \% \text { (diplopia, } \\
\text { facial } \\
\text { dysesthesia, } \\
\text { vertigo, ataxia) }\end{array}$ \\
\hline $\begin{array}{l}\text { Kano et al., } \\
2018 \text { [5] }\end{array}$ & 92 & $\begin{array}{l}\text { SRS by } \\
\text { Yknife }\end{array}$ & $I X-X \mid$ & $\begin{array}{l}51 \text { mos } \\
\text { median }\end{array}$ & $\begin{array}{l}93 \% \\
(3 y) \\
82 \% \\
(10 y)\end{array}$ & $\begin{array}{l}8 \% \text { (details not } \\
\text { written in the } \\
\text { abstract) }\end{array}$ \\
\hline $\begin{array}{l}\text { Shinya et al., } \\
2021 \text { [16] }\end{array}$ & 54 & $\begin{array}{l}\text { SRS by } \\
\text { yknife }\end{array}$ & $\begin{array}{l}\text { V (22), VII (7), IX- } \\
\text { XI (25) }\end{array}$ & $\begin{array}{l}72 \text { mos } \\
\text { mean }\end{array}$ & $98 \%$ & $\begin{array}{l}\text { Hydrocephalus } \\
(4 \%) \text {, lower } \\
\text { cranial nerve } \\
\text { palsy }(2 \%)\end{array}$ \\
\hline $\begin{array}{l}\text { Langlois et al., } \\
2021 \text { [6] }\end{array}$ & 25 & $\begin{array}{l}\text { SRS by } \\
\text { yknife }\end{array}$ & $\begin{array}{l}\text { III (11), IV (11), VI } \\
(3)\end{array}$ & $\begin{array}{l}41 \text { mos } \\
\text { median }\end{array}$ & $92 \%$ & $\begin{array}{l}\text { Cystic tumor } \\
\text { change (4\%) }\end{array}$ \\
\hline Our study & 45 & $\begin{array}{l}\text { hSRT by } \\
\text { CyberKnife }\end{array}$ & $\begin{array}{l}\text { V(12), VI (1), VII } \\
\text { (3), IX-XI (13), XII } \\
\text { (8), spine (6), } \\
\text { parapharyngeal } \\
\text { space (2) }\end{array}$ & $\begin{array}{l}74 \text { mos } \\
\text { mean, } \\
75 \text { mos } \\
\text { median }\end{array}$ & $96 \%$ & $\begin{array}{l}\text { Transient mass } \\
\text { effect due to } \\
\text { transient tumor } \\
\text { expansion (22\%), } \\
\text { dysgeusia (4\%), } \\
\text { tinnitus (2\%) }\end{array}$ \\
\hline $\begin{array}{l}\text { LCR: local cor } \\
\text { hypofractiona }\end{array}$ & $e, y:$ & nos: mc & $\begin{array}{l}\text { SRT: conventic } \\
\text { tor }\end{array}$ & $T, f S$ & $a c$ & d SRT, hSRT: \\
\hline
\end{tabular}

\section{Good local control rate of both intra/ extracranial tumors}

In our cohort, except for spinal schwannomas, 17 patients (38\%) had extracranial tumor portion (Table 3). We showed that CyberKnife could treat the extracranial tumor segment as effectively as the intracranial segment (Table 4). Two past studies on jugular foramen schwannomas reported that dumbbell-type tumors and large tumor volume as risk factors for tumor control failure.[4, 5] The cohorts of these studies were treated by gamma knife SRS. In our cohort, all tumors were treated by CyberKnife SRT, and all tumors regardless of portions of intra and extracranial space were controlled. This difference is attributed to the difference in way of radiation delivery between gamma knife and CyberKnife. Gamma knife is more suitable for intracranial targets whereas CyberKnife is suitable for extracranial targets as well as intracranial targets. Since both the dumbbell-shaped schwannomas and primary extracranial schwannomas were effectively treated (Tables 3 and 4), these tumors may be more suited for CyberKnife. Having said that, we still need to accumulate evidence as we have few studies on treatment outcomes of these tumors performed by CyberKnife (Table 5). 
As for clinical symptoms, our data showed older age and previous surgical history were statistically significant risk factors against symptom improvement (Supplementary Table 2). Based on this, surgical resection, if chosen, should be pursued under the top priority of not damaging the surrounding nerves, especially in older patients. However, since our center is a referral center and whether to proceed with upfront SRT or adjuvant SRT was not decided based on the strict criteria, our data was at risk for selection bias.

\section{Transient tumor expansion (TTE)}

In our study, the rate of ARE was higher than in previous studies. Although only $4 \%$ of the patients resulted in permanent ARE, as much as $25 \%$ (eight out of 32 patients) experienced worsening of clinical symptoms due to TTE. TTE itself occurred in $41 \%$ (13 out of 32 patients). Although this rate is high, it is in the range of previously reported rates between 17 to $74 \%$.[9] Patients should be well-informed about this phenomenon to relieve the distress and anxiety caused by this if happened. Although none of the patients in our cohort chose surgical intervention to relieve the mass effect caused by transient tumor expansion, surgical resection of the tumor is an option as well as supportive care.

\section{Limitations}

The limitations were the retrospective nature of the study and that this study was carried out in a single institution. Since our institution was a referral center for SRT, incomplete clinical and radiological information was available. These may have affected the exact rate of ARE and TTE and our data was at risk of selection bias.

\section{Conclusions}

Hypofractionated SRT is a valid modality for head/ neck/ spine non-vestibular schwannomas over the long term and CyberKnife-based SRT effectively treats schwannomas of dumbbell-shaped as well as those located in the extracranial space. However, some patients experience symptoms from transient tumor expansion.

\section{Declarations}

Funding: Not applicable.

Conflicts of interest/Competing interests:Not applicable.

Availability of data and material: Data transparency was confirmed.

Code availability: Not applicable

Ethical approval: This study was done under our institutional review board's approval and did not require patient consent. 
Consent to participate: Our institutional review board did not require informed consent for study participation because this study relied on information obtained as part of routine clinical practice.

Consent for publication: Our institutional review board did not require informed consent for study publication because the publication data were de-identified as for the information obtained.

Authors' contributions: All authors read and approved the final manuscript. SH made a study design, collected patient data, drafted and revised the manuscript. KK, KS, RN, and SI were the supervisors.

\section{References}

1. Choi CY, Soltys SG, Gibbs IC, Harsh GR, Sakamoto GT, Patel DA, Lieberson RE, Chang SD, Adler JR (2011) Stereotactic radiosurgery of cranial nonvestibular schwannomas: results of single- and multisession radiosurgery. Neurosurgery 68:1200-1208. discussion 1208 doi:10.1227/NEU.0b013e31820c0474

2. Elsharkawy M, Xu Z, Schlesinger D, Sheehan JP (2012) Gamma Knife surgery for nonvestibular schwannomas: radiological and clinical outcomes. J Neurosurg 116:66-72. doi:10.3171/2011.8.Jns11215

3. Hamm KD, Gross MW, Fahrig A, Surber G, Henzel M, Kleinert G, Grabenbauer GG, Engenhart-Cabillic R (2008) Stereotactic radiotherapy for the treatment of nonacoustic schwannomas. Neurosurgery 62:A29-36. discussion A36 doi:10.1227/01.neu.0000325934.16229.03

4. Hasegawa T, Kato T, Kida Y, Sasaki A, Iwai Y, Kondoh T, Tsugawa T, Sato M, Sato M, Nagano O, Nakaya K, Nakazaki K, Kano T, Hasui K, Nagatomo Y, Yasuda S, Moriki A, Serizawa T, Osano S, Inoue A (2016) Gamma Knife surgery for patients with jugular foramen schwannomas: a multiinstitutional retrospective study in Japan. J Neurosurg 125:822-831. doi:10.3171/2015.8.Jns151156

5. Kano H, Meola A, Yang HC, Guo WY, Martínez-Alvarez R, Martínez-Moreno N, Urgosik D, Liscak R, Cohen-Inbar O, Sheehan J, Lee JYK, Abbassy M, Barnett GH, Mathieu D, Kondziolka D, Lunsford LD (2018) Stereotactic radiosurgery for jugular foramen schwannomas: an international multicenter study. J Neurosurg 129:928-936. doi:10.3171/2017.5.Jns162894

6. Langlois AM, lorio-Morin C, Faramand A, Niranjan A, Lunsford LD, Mohammed N, Sheehan JP, Liščák R, Urgošík D, Kondziolka D, Lee CC, Yang HC, Atik AF, Mathieu D (2021) Outcomes after stereotactic radiosurgery for schwannomas of the oculomotor, trochlear, and abducens nerves. J Neurosurg 1-7. doi:10.3171/2020.8.Jns20887

7. Langlois AM, lorio-Morin C, Masson-Côté L, Mathieu D (2018) Gamma Knife Stereotactic Radiosurgery for Nonvestibular Cranial Nerve Schwannomas. World Neurosurg 110:e1031-e1039. doi:10.1016/j.wneu.2017.11.163

8. Martin JJ, Kondziolka D, Flickinger JC, Mathieu D, Niranjan A, Lunsford LD (2007) Cranial nerve preservation and outcomes after stereotactic radiosurgery for jugular foramen schwannomas. Neurosurgery 61:76-81. discussion 81 doi:10.1227/01.neu.0000279726.90650.6d 
9. Nagano O, Higuchi Y, Serizawa T, Ono J, Matsuda S, Yamakami I, Saeki N (2008) Transient expansion of vestibular schwannoma following stereotactic radiosurgery. J Neurosurg 109:811-816. doi:10.3171/jns/2008/109/11/0811

10. National Cancer I (2010) Common terminology criteria for adverse events: (CTCAE)

11. Pan L, Wang EM, Zhang N, Zhou LF, Wang BJ, Dong YF, Dai JZ, Cai PW (2005) Long-term results of Leksell gamma knife surgery for trigeminal schwannomas. J Neurosurg 102 Suppl 220-224. doi:10.3171/jns.2005.102.s_supplement.0220

12. Puataweepong $P$, Dhanachai $M$, Hansasuta $A$, Saetia K, Dangprasert $S$, Sitathanee $C$, Yongvithisatid $P$ (2016) Clinical Outcomes of Intracranial Nonvestibular Schwannomas Treated with LinacBased Stereotactic Radiosurgery and Radiotherapy. Asian Pacific journal of cancer prevention: APJCP 17:3271-3276

13. Sachdev S, Dodd RL, Chang SD, Soltys SG, Adler JR, Luxton G, Choi CY, Tupper L, Gibbs IC (2011) Stereotactic radiosurgery yields long-term control for benign intradural, extramedullary spinal tumors. Neurosurgery 69:533-539. discussion 539 doi:10.1227/NEU.0b013e318218db23

14. Sheehan J, Yen CP, Arkha Y, Schlesinger D, Steiner L (2007) Gamma knife surgery for trigeminal schwannoma. J Neurosurg 106:839-845. doi:10.3171/jns.2007.106.5.839

15. Shin DW, Sohn MJ, Kim HS, Lee DJ, Jeon SR, Hwang YJ, Jho EH (2015) Clinical analysis of spinal stereotactic radiosurgery in the treatment of neurogenic tumors. Journal of neurosurgery Spine 23:429-437. doi:10.3171/2015.1.Spine 14910

16. Shinya Y, Hasegawa H, Shin M, Sugiyama T, Kawashima M, Katano A, Kashio A, Kondo K, Saito N (2021) Long-Term Outcomes of Stereotactic Radiosurgery for Trigeminal, Facial, and Jugular Foramen Schwannoma in Comparison with Vestibular Schwannoma. Cancers 13:1140. doi: $10.3390 /$ cancers 13051140

17. Showalter TN, Werner-Wasik M, Curran WJ Jr, Friedman DP, Xu X, Andrews DW (2008) Stereotactic radiosurgery and fractionated stereotactic radiotherapy for the treatment of nonacoustic cranial nerve schwannomas. Neurosurgery 63:734-740. discussion 740 doi:10.1227/01.Neu.0000325496.10148.B3

\section{Figures}



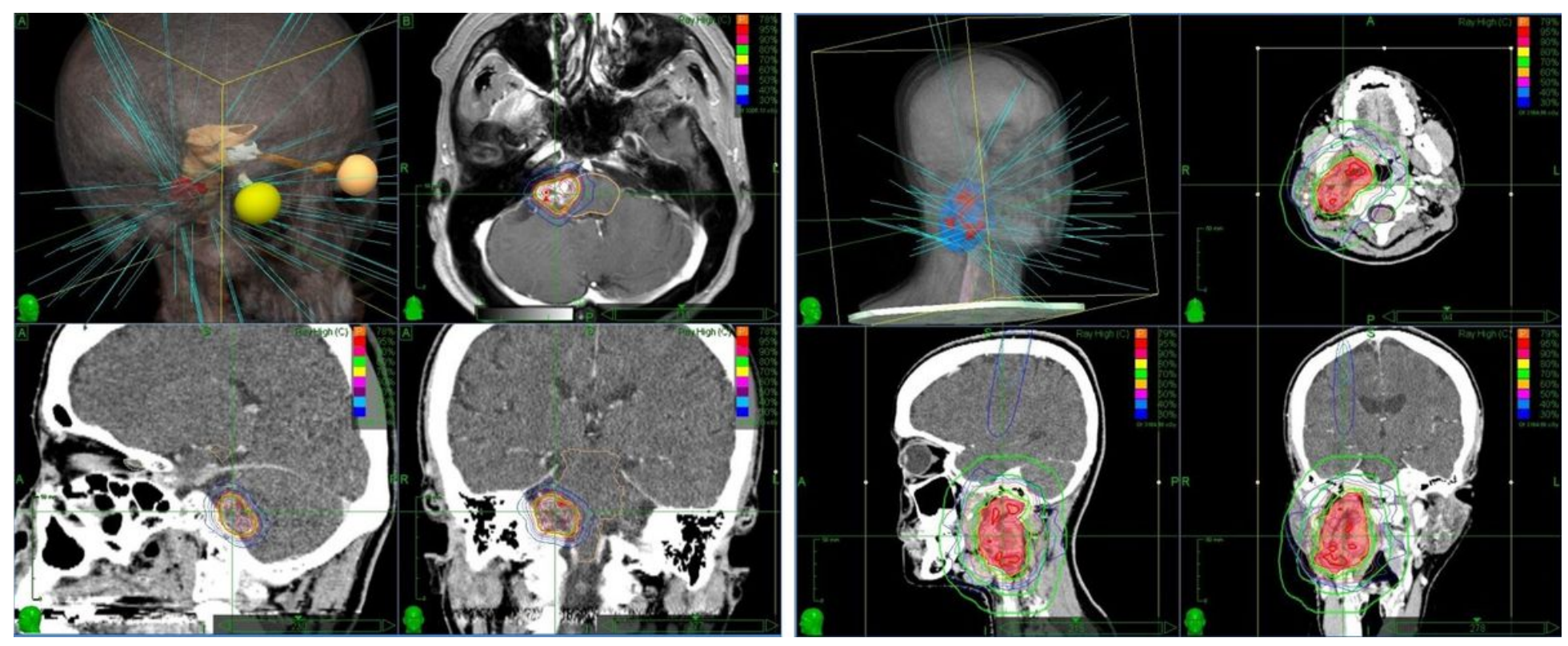

\section{Figure 1}

Two illustrative cases of stereotactic radiotherapy (SRT). A 62-year-old female was referred after an open tumor biopsy for jugular foramen schwannoma (primary intracranial). Since she had had no symptoms the operation was finished after obtaining the pathological diagnosis. We performed SRT with the prescribed dose covering $95 \%$ of the planning target volume (D95) of 25Gy in 5 fractions for target tumor volume of 3cc. The therapy resulted in transient tumor enlargement (TTE), and permanent common terminology criteria for adverse events (CTCEA) grade 1 dysgeusia and CTCAE grade 1 tinnitus. She has been stable radiologically and clinically after 101 and 105 months, respectively (Left). A 30-year-old female presented with dysphagia and was diagnosed with parapharyngeal space schwannoma (primary extracranial) after a biopsy. Upfront hSRT was performed with D95 of 25Gy in 5 fractions for a target tumor volume of $55 \mathrm{cc}$. After TTE, her dysphagia improved and the tumor shrank. She has been stable radiologically and clinically after 92 months.

\section{Supplementary Files}

This is a list of supplementary files associated with this preprint. Click to download.

- SupplementaryTable.docx 